\title{
Robust estimation of scale of an exponential distribution
}

\author{
U. Gather ${ }^{1}$ and V. Schultze \\ Department of Statistics, University of Dortmund, Vogelpothsweg 87, D-44221 \\ Dortmund, Federal Republic of Germany
}

We consider a new estimator of scale for exponential samples which is most B-robust in the sense of Hampel et al. (1986). This estimator is compared with two other estimators which were proposed by Rousseeuw and Croux (1993) but for a Gaussian model. All three estimators have the same breakdown point, but their explosion bias curves are different. It is shown that under a gross error model the explosion bias curve of the new estimator performs better than the bias curves of the other estimators.

Key Words \& Phrases: Influence function, breakdown point, explosion bias curve

\section{Introduction}

Often, when some kind of non-aging property of a positive random variable can be relied upon, it is assumed that i. i. d. observations $x_{1}, \ldots, x_{N}>0$ of this variable come from an exponential distribution with unknown scale parameter $\nu>0$. Thus, we have a likelihood

$$
f_{\nu}\left(x_{1}, \ldots, x_{N}\right):=\left(\frac{1}{\nu}\right)^{N} \exp \left(-\frac{1}{\nu} \sum_{i=1}^{N} x_{i}\right), \quad x_{1}, \ldots, x_{N}>0 .
$$

Consider the unknown scale parameter $\nu$. Any scale estimator $S_{N}$ should be scale equivariant, i. e.:

$$
S_{N}\left(a x_{1}, \ldots, a x_{N}\right)=a S_{N}\left(x_{1}, \ldots, x_{N}\right) \text { for all } a>0 .
$$

Most popular is the maximum likelihood estimator

$$
\hat{\nu}_{M L}\left(x_{1}, \ldots, x_{N}\right)=\frac{1}{N} \sum_{i=1}^{N} x_{i}
$$

\footnotetext{
${ }^{1}$ gather@omega.statistik.uni-dortmund.de
} 
because it is asymptotically efficient. But $\hat{\nu}_{M L}$ can be bad, if the sample is contaminated. For instance one extremely large outlier in the sample suffices to make $\hat{\nu}_{M L}$ arbitrarily large. In this case it is often said that "the estimator has broken down".

Therefore, the aim of this paper is to introduce and to investigate some new robust estimators for the unknown scale parameter $\nu>0$ of an exponential distribution, which can deal with such "bad" observations. Our paper is organized as follows: In chapter 2, a new estimator is defined, which is a standardized version of the sample median. Its robustness properties are investigated and it is shown that its gross-error-sensitivity is minimal. As the price for this optimality property is a low asymptotic efficiency, other robust estimators (RousseEuW AND CROUX, 1993) are considered in the third chapter. Finally, we compare the maximum asymptotic biases of the estimators presented here.

\section{The standardized median}

M-estimators are very popular and well investigated candidates for robust estimators. An M-estimator is defined as solution of the equation

$$
\sum_{i=1}^{N} \psi\left(x_{i}, S_{N}\right)=0
$$

with some function $\psi(x ; \nu)$. An M-estimator of scale should fulfill $\psi(x, \nu)=\psi\left(\frac{x}{\nu}\right)$. If $G_{N}$ denotes the empirical distribution function generated by the sample, then a solution $S_{N}$ of (??) can be expressed as $S\left(G_{N}\right)$, where $S$ is a functional given by

$$
\int \psi(x, S(G)) d G(x)=0
$$

The M-estimator corresponding to $\tilde{\psi}(x, \nu):=\operatorname{sign}(x-\nu \log 2)$ is

$$
S M_{N}\left(x_{1}, \ldots, x_{N}\right):=\frac{1}{\log 2} \operatorname{Med}\left(x_{1}, \ldots, x_{N}\right) .
$$

To ensure Fisher-consistency of the median in independent identically exponentially distributed samples, the sample median must be multiplied by $[\log 2]^{-1}$. The resulting estimate is called the standardized median and denoted by $S M$. Some robustness properties of the standardized median can be investigated by means of the influence function (HAMPEL, 1974). To begin with, the functional version of the standardized median is defined as

$$
S M(G):=\frac{1}{\log 2} G^{-1}\left(\frac{1}{2}\right), \quad \text { where } G \text { is a distribution on }\left(\mathcal{R}^{+}, \mathbf{B}\right) .
$$


The influence function of the standardized median is equal to (STAUDTE AND SHEATER, 1990, p. 59)

$$
I F\left(x ; S M, F_{\nu}\right)= \begin{cases}-\frac{\nu}{\log 2} & x<\nu \log 2 \\ 0 & x=\nu \log 2 \\ \frac{\nu}{\log 2} & x>\nu \log 2\end{cases}
$$

with gross-error sensitivity

$$
\gamma^{*}\left(S M, F_{\nu}\right):=\sup _{x \in \mathcal{R}^{+}}\left|I F\left(x ; S M, F_{\nu}\right)\right|=\frac{\nu}{\log 2} .
$$

The asymptotic variance (STAUDTE AND SHEATER, 1990, p. 64) can be expressed as

$$
V\left(S M, F_{\nu}\right)=\frac{1}{\left(2(\log 2) f_{\nu}(\nu \log 2)\right)^{2}}=2.0814 \nu^{2} .
$$

In general, an estimator with limited gross-error-sensitivity is called B-robust. An estimator with minimal gross-error sensitivity is called most B-robust (HAMPEL ET AL., 1986, p. 133). The following theorem shows that for estimating the scale paramter $\nu$ of an exponential distribution the standardized median has smallest possible gross-error sensitivity.

Theorem 2.1. Let $\Psi_{\text {exp }}$ be the class of all functions $\psi: \mathcal{R}^{+} \rightarrow \mathcal{R}$ satisfying:

(i) The function $\psi$ is well-defined and continuous on $\mathcal{R}^{+}$except for a finite set $C(\psi)$. In each point of $C(\psi)$, there exist finite left and right limits of $\psi$ which are different.

(ii) $\int_{0}^{\infty} \psi\left(\frac{y}{\nu}\right) d F_{\nu}(y)=0$ for all $\nu>0$.

Let $S_{N}$ be an M-estimator corresponding to $\psi \in \Psi_{\exp }$ and $S(\psi)$ be the corres ponding functional then

$$
\gamma^{*}\left(S(\psi), F_{\nu}\right) \geq \frac{\nu}{\log 2} \text { for all } \psi \in \Psi_{\text {exp }} \text { and } F_{\nu} \in \mathcal{F}_{\text {exp }}
$$

\section{Proof.}

$$
\begin{aligned}
\gamma^{*}\left(S(\psi), F_{\nu}\right) & =\sup _{x \in \mathcal{R}^{+}}\left|\frac{\psi\left(\frac{x}{\nu}\right)}{\int_{0}^{\infty} \psi\left(\frac{y}{\nu}\right) s(y, \nu) f_{\nu}(y) d y}\right| \\
& =\frac{\sup _{x \in \mathcal{R}^{+}}\left|\psi\left(\frac{x}{\nu}\right)\right|}{\left|\int_{0}^{\infty} \psi\left(\frac{y}{\nu}\right)\left(\frac{y}{\nu}-1\right) \frac{1}{\nu^{2}} e^{-\frac{y}{\nu}} d y\right|} \\
& =\frac{\sup _{x \in \mathcal{R}^{+}}\left|\psi\left(\frac{x}{\nu}\right)\right|}{\left|\int_{0}^{\infty} \psi\left(\frac{y}{\nu}\right)\left(\frac{y}{\nu}-\frac{t}{\nu}\right) \frac{1}{\nu^{2}} e^{-\frac{y}{\nu}} d y\right|} \\
& \geq\left(\int_{0}^{\infty}\left|\frac{y}{\nu}-\frac{t}{\nu}\right| \frac{1}{\nu^{2}} e^{-\frac{y}{\nu}} d y\right)^{-1} \\
& =\left(\frac{t}{\nu^{2}}+\frac{2}{\nu} e^{-\frac{t}{\nu}}-\frac{1}{\nu}\right)^{-1}=: w(t)
\end{aligned}
$$


and $w(t) \leq w(\nu \log 2)=\frac{\nu}{\log 2}$ for all $t>0$.

In order to find the maximum number of "bad" observations, the standardized median can deal with, a replacement version of the finite sample breakdown point of an estimator is used (RousseEuW AND LEROY, 1988). The breakdown point $\epsilon_{N}^{*}\left(S_{N}, \underline{x}_{N}\right)$ of $S_{N}$ is defined as

$$
\epsilon_{N}^{*}\left(S_{N}, \underline{x}_{N}\right):=\min \left\{\frac{m}{N} ; \sup _{\underline{\underline{x}}_{N}} S_{N}\left(\underline{\tilde{x}}_{N}\right)=\infty \text { or } \inf _{\underline{\tilde{x}}_{N}} S_{N}\left(\underline{\tilde{x}}_{N}\right)=0\right\},
$$

where $\underline{\tilde{x}}_{N}$ is obtained from $\underline{x}_{N}$ by replacing $\mathrm{m}$ values by arbitrarily chosen ones.

Theorem 2.2. The finite sample breakdown point of the standardized median is equal to

$$
\epsilon_{N}^{*}\left(S M_{N}, \underline{x}_{N}\right)=\frac{\left\lceil\frac{N}{2}\right\rceil}{N}
$$

and for the breakdown point $\epsilon^{*}\left(S M, F_{\nu}\right)$ itself, we have

$$
\epsilon^{*}\left(S M, F_{\nu}\right)=\lim _{N \rightarrow \infty} \epsilon_{N}^{*}\left(S M_{N}, \underline{x}_{N}\right)=\frac{1}{2} .
$$

Proof. Let $0<x_{(1)} \leq \ldots \leq x_{(n)}$ denote the ordered sample. If $x_{\left(\left\lfloor\frac{N}{2}+1\right\rfloor\right)}, \ldots, x_{(N)}$ are replaced by an arbitrary $z \in \mathcal{R}^{+}$, then $\underline{\underline{x}}_{N}:=\left\{x_{(1)}, \ldots, x_{\left(\left\lfloor\frac{N}{2}\right\rfloor\right)}, z, \ldots, z\right\}$ and $S M_{N}\left(\underline{\tilde{x}}_{N}\right)$ converges to $\infty$ for $N$ tending to $\infty$. Hence,

$$
\epsilon_{N}^{*}\left(S M_{N}, \underline{x}_{N}\right) \leq \frac{\left\lceil\frac{N}{2}\right\rceil}{N} .
$$

If $x_{\left(\left\lfloor\frac{N}{2}\right\rfloor\right)}, \ldots, x_{(N)}$ are replaced by an arbitrary $z \in \mathcal{R}^{+}$, then

$$
\underline{\breve{x}}_{N}:=\left\{x_{(1)}, \ldots, x_{\left(\left\lfloor\frac{N}{2}-1\right\rfloor\right)}, z, \ldots, z\right\} \text { and } S M_{N}\left(\underline{\breve{x}}_{N}\right)=S M_{N}\left(\underline{x}_{N}\right) .
$$

Hence,

$$
\epsilon_{N}^{*}\left(S M_{N}, \underline{x}_{N}\right) \geq \frac{\left\lceil\frac{N}{2}\right\rceil}{N}
$$

Rousseeuw And Croux (1993) also consider the behaviour of robust estimators under a so called gross error model (HuBER, p. 11). Let

$$
\mathcal{F}_{\epsilon}:=\left\{G \in \mathcal{M}^{1} \mid G=(1-\epsilon) F_{\nu}+\epsilon H_{\text {pos }}\right\}, \quad \epsilon>0,
$$


with

$$
H_{\text {pos }} \in\left\{H \in \mathcal{M}^{1} \mid H(0)=0\right\} .
$$

The explosion bias curve of the functional $S$ in $F_{\nu}$ is defined as

$$
B^{\epsilon}\left(S, F_{\nu}\right):=\sup _{G \in \mathcal{F}_{\epsilon}} S(G) .
$$

There exist several connections between the explosion bias curve, the gross-error sensitivity and the breakdown point (HAMPEL ET AL., 1986, p. 177):

(i) If the explosion bias curve $B^{\epsilon}\left(S, F_{\nu}\right)$, plotted as a function of the mixture proportion $\epsilon$ of contamination, is differentiable at $\epsilon=0$, then the slope of the tangent at 0 is the gross-error sensitivity $\gamma^{*}\left(S, F_{\nu}\right)$.

(ii) The breakdown point $\epsilon^{*}\left(S, F_{\nu}\right)$ is given by

$$
\epsilon^{*}\left(S, F_{\nu}\right)=\min _{\epsilon \in\left(0, \frac{1}{2}\right)}\left\{B^{\epsilon}\left(S, F_{\nu}\right)=\infty\right\}
$$

(DONOHO AND LiU 1988).

Theorem 2.3. If $\epsilon \in\left(0, \frac{1}{2}\right)$, then

$$
B^{\epsilon}\left(S M, F_{\nu}\right)=\nu \frac{\log \frac{2(1-\epsilon)}{1-2 \epsilon}}{\log 2} .
$$

Proof. Let $\epsilon$ be any value in $\left(0, \frac{1}{2}\right)$. Because the standardized median $S M_{N}$ is monotone in each observation $x_{i}$, it follows that

$$
B^{\epsilon}\left(S M, F_{\nu}\right)=\lim _{N \rightarrow \infty} S M\left(G_{x_{N}}\right)
$$

with

$$
G_{x_{N}}:=(1-\epsilon) F_{\nu}+\epsilon \Delta_{x_{N}} \text { and } \lim _{N \rightarrow \infty} x_{N}=\infty .
$$

Substituting $G_{x_{N}}$ yields

$$
B^{\epsilon}\left(S M, F_{\nu}\right)=\frac{1}{\log 2} \lim _{x_{N} \rightarrow \infty}\left[(1-\epsilon) F_{\nu}+\epsilon \Delta_{x_{N}}\right]^{-1}\left(\frac{1}{2}\right) .
$$

Without loss of generality let $x_{N}>\nu \log 2$. Then independently of $\mathrm{N}$,

$$
\left[(1-\epsilon) F_{\nu}+\epsilon \Delta_{x_{N}}\right]^{-1}\left(\frac{1}{2}\right)=\nu \log \frac{2(1-\epsilon)}{1-2 \epsilon} .
$$

Figure 1 shows the explosion bias curve of the standardized median. The minimum slope of the tangent at $\epsilon=0$ reflects, that $S M_{N}\left(x_{1}, \ldots, x_{N}\right)$ is a most B-robust estimator. The value of $\epsilon$ for which the explosion bias curve becomes infinite is the breakdown point $\epsilon^{*}$. 


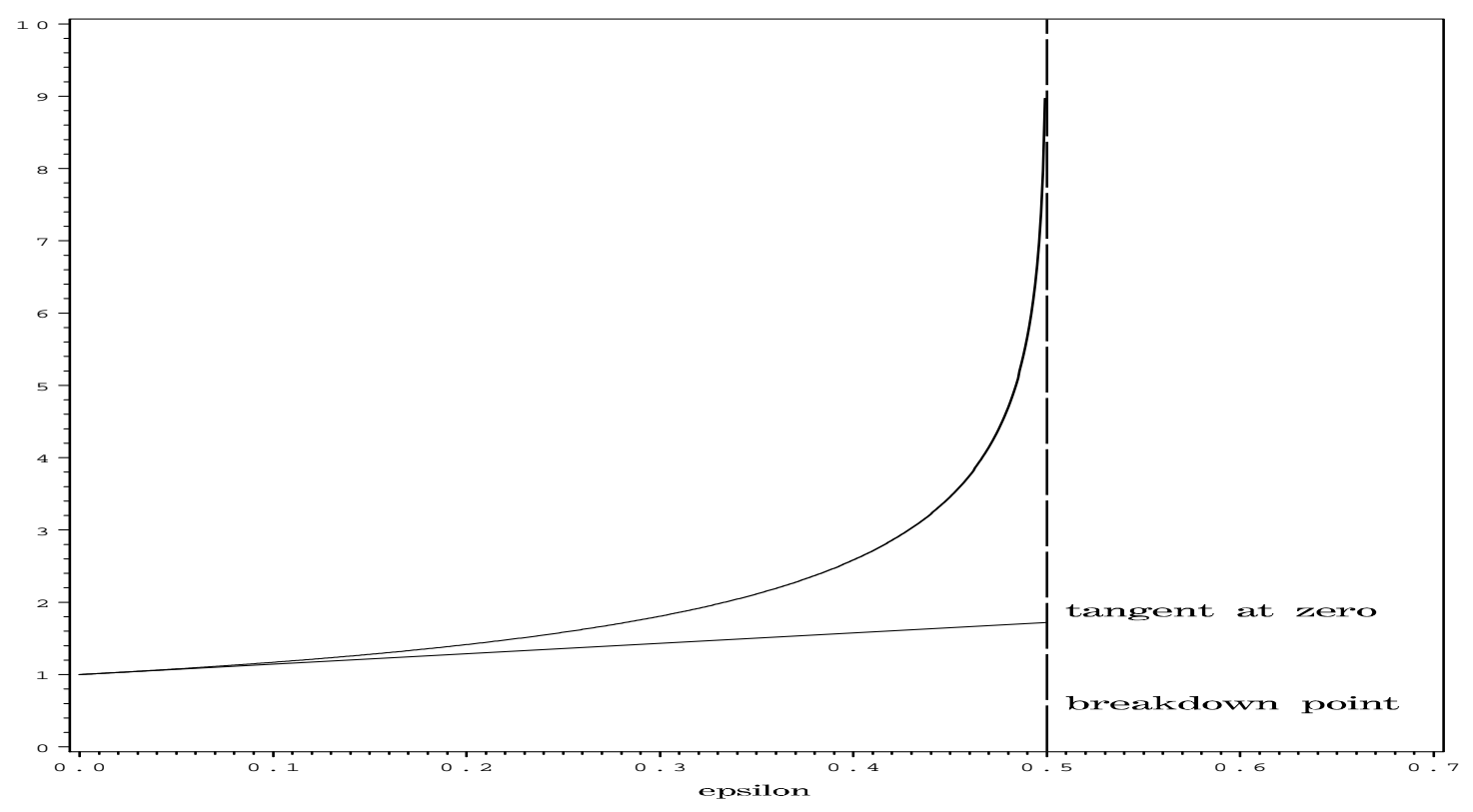

Figure 1: Explosion bias curve of the standardized median with $\nu=1$.

\section{The RCS- and the Q-estimator}

The drawback of the standardized median is that it has a small asymptotic relative efficiency (ARE) at the exponential distributions of only 48\%. Therefore, two alternatives, which are of the same type of those proposed by RoUsSEEUW AND Croux (1993) to estimate the variance in normal samples and which work well in this situation, are tried out for the exponential case, too. The first is an estimator which Rousseeuw And Croux (1993) called "S-estimator". To distinguish between the "S-estimator" and the general class of S-estimators (RoussEEuW AND YOHAI, 1984), this estimator is called RCS-estimator here, it is given by

$$
\begin{array}{rll}
R C S\left(x_{1}, \ldots, x_{N}\right) \quad:= & 1 & .6982 M_{i}\left\{\operatorname{Med}_{j}\left\{\left|x_{i}-x_{j}\right|\right\}\right\} \\
\text { with } & x_{i}, x_{j}>0 \text { and } i, j \in\{1, \ldots, N\} .
\end{array}
$$

The constant 1.6982 ensures Fisher-consistency of the RCS-estimator for samples from an exponential distribution. The asymptotic version of the RCS-estimator is defined as follows: Let $G \in \mathcal{M}^{1}, X, Y$ be stochastically independent with $X \sim G, Y \sim G$ and

$$
g_{G}(x):=\operatorname{Med}_{Y}|x-Y|, \quad x>0 .
$$

Then the functional $R C S(G)$ is equal to

$$
\operatorname{RCS}(G)=1.6982 \text { Medx } g_{G}(X) \text {. }
$$


It can be shown (RousseEuw AND CROUX, 1993) for the gross-error sensitivity

$$
\gamma^{*}\left(R C S, F_{\nu}\right)=1.8447 \nu
$$

the asymptotic variance equals

$$
V\left(R C S, F_{\nu}\right)=1.8217 \nu^{2}
$$

and the asymptotic relative efficiency of the RCS-estimator at exponential distributions is $55 \%$. In these respects the RCS-estimator is comparable with the $20 \%$-trimmed mean $\bar{x}_{20}$.

More exactly the gross-error sensitivity of the trimmed mean is $\gamma^{*}\left(\bar{x}_{20}, F_{\nu}\right)=1.72$ and the asymptotic relative efficiency is $A R E\left(\bar{x}_{20}, \hat{\nu}_{M L}\right)=0.72$ (KIMBER, 1983), but its breakdown point is $\epsilon^{*}\left(\bar{x}_{20}, F_{\nu}\right)=0.2$ which indeed is quite smaller than the breakdown point of the RCS-estimator, which is equal to 0.5 .

Theorem 3.1. The explosion bias curve of the RCS-estimator is

$$
B^{\epsilon}\left(R C S, F_{\nu}\right)=1.6982 \nu x_{0}(\epsilon)
$$

where $x_{0}(\epsilon)$ is the smallest positive solution of

$$
(1-2 \epsilon)\left(e^{2 x_{0}(\epsilon)}-1\right)-\left(e^{x_{0}(\epsilon)}-e^{-x_{0}(\epsilon)}\right)-1 \geq 0 .
$$

Proof. Let $\epsilon$ be any value in $\left(0, \frac{1}{2}\right)$. First consider $F_{1}(x)=1-e^{-x}, x>0$, then

$$
B^{\epsilon}\left(R C S, F_{1}\right)=\lim _{N \rightarrow \infty} \operatorname{RCS}\left(G_{x_{N}}\right)
$$

with $G_{x_{N}}:=(1-\epsilon) F_{1}+\epsilon \Delta_{x_{N}}$ and $\lim _{N \rightarrow \infty} x_{N}=\infty$. Let $X \sim G_{x_{N}}$ and $Y \sim G_{x_{N}}$. Then $g_{G_{x_{N}}}(x)$ is the smallest positive value for which

$$
P\left(|Y-x| \leq g_{G_{x_{N}}}(x)\right) \geq \frac{1}{2}
$$

or equivalently

$$
G_{x_{N}}\left(x+g_{G_{x_{N}}}(x)\right)-G_{x_{N}}\left(x-g_{G_{x_{N}}}(x)\right)+P\left(Y=x-g_{G_{x_{N}}}(x)\right) \geq \frac{1}{2} .
$$

Substituting $G_{x_{N}}$ yields

$$
\begin{aligned}
&(1-\epsilon)\left\{F_{1}\left(x+g_{G_{x_{N}}}(x)\right)-\right.\left.F_{1}\left(x-g_{G_{x_{N}}}(x)\right)\right\} \\
&+\epsilon\left\{\Delta_{x_{N}}\left(x+g_{G_{x_{N}}}(x)\right)-\Delta_{x_{N}}\left(x-g_{G_{x_{N}}}(x)\right)\right\} \\
&+\epsilon 1_{\left(x-g_{G_{x_{N}}}(x)=x_{N}\right)} \geq \frac{1}{2} .
\end{aligned}
$$


With $\epsilon<\frac{1}{2}$, for each $M>0$ there exists $N_{0}$ such that for all $N>N_{0}$

$$
\sup _{x<M}\left(x+g_{G_{x_{N}}}(x)\right)<x_{N},
$$

because if $N$ is large enough, then

$$
\begin{aligned}
\sup _{x<M}\left[x+g_{G_{x_{N}}}(x)\right] & \leq \sup _{x<M}\left[x+\operatorname{Med}_{Y}|Y-x|\right] \\
& \leq 2 M+\operatorname{Med}_{Y}(Y) \leq x_{N}
\end{aligned}
$$

This means that $g_{G_{x_{N}}}$ is the solution of

$$
\begin{aligned}
(1-\epsilon)\left[F_{1}\left(x+g_{G_{x_{N}}}(x)\right)-F_{1}\left(x-g_{G_{x_{N}}}(x)\right)\right] & =\frac{1}{2} \quad \text { if } x \geq g_{G_{x_{N}}}(x) \\
\text { and }(1-\epsilon) F_{1}\left(x+g_{G_{x_{N}}}(x)\right) & =\frac{1}{2} \quad \text { if } x \leq g_{G_{x_{N}}}(x)
\end{aligned}
$$

which leads to

$$
g_{G_{x_{N}}}(x)= \begin{cases}g_{u}(x) & x \leq-\log \sqrt{\frac{1-2 \epsilon}{2(1-\epsilon)}} \\ g_{o}(x) & x \geq-\log \sqrt{\frac{1-2 \epsilon}{2(1-\epsilon)}}\end{cases}
$$

with

$$
\begin{aligned}
g_{u}(x) & :=-x-\log \left[\frac{1-2 \epsilon}{2(1-\epsilon)}\right] \\
g_{o}(x) & :=\log \left[\frac{e^{x}}{4(1-\epsilon)}+\sqrt{\frac{e^{2 x}}{16(1-\epsilon)^{2}}+1}\right]
\end{aligned}
$$

and

$$
\begin{aligned}
g_{u}^{-1}(x) & =-x-\log \left[\frac{1-2 \epsilon}{2(1-\epsilon)}\right], \\
g_{o}^{-1}(x) & =\log \left[2(1-\epsilon)\left(e^{x}-e^{-x}\right)\right] .
\end{aligned}
$$

This implies that

$$
B^{\epsilon}\left(R C S, F_{1}\right)=1.6982 \lim _{x_{N} \rightarrow \infty}\left[\operatorname{Med}_{X} g_{G_{x_{N}}}(X)\right] .
$$

Let $x_{0}^{N}(\epsilon):=\operatorname{Med}_{X} g_{G_{x_{N}}}$. Then $x_{0}^{N}(\epsilon)$ is the smallest positive value for which

$$
P\left(g_{G_{x_{N}}}(X) \leq x_{0}^{N}(\epsilon)\right) \geq \frac{1}{2}
$$

If $M(\epsilon)$ is chosen such that $x_{0}^{N}(\epsilon)<M$, then

$$
P\left(X \leq g_{o}^{-1}\left(x_{o}^{N}(\epsilon)\right)\right)-P\left(X \leq g_{u}^{-1}\left(x_{u}^{N}(\epsilon)\right)\right)+P\left(X=g_{u}^{-1}\left(x_{o}^{N}(\epsilon)\right)\right) \geq \frac{1}{2}
$$


or equivalently

$$
\begin{gathered}
(1-\epsilon) F_{1}\left(g_{o}^{-1}\left(x_{0}^{N}(\epsilon)\right)\right)+\epsilon \Delta_{x_{N}}\left(g_{o}^{-1}\left(x_{0}^{N}(\epsilon)\right)\right) \\
-(1-\epsilon) F_{1}\left(g_{u}^{-1}\left(x_{0}^{N}(\epsilon)\right)\right)-\epsilon \Delta_{x_{N}}\left(g_{u}^{-1}\left(x_{0}^{N}(\epsilon)\right)\right)+\epsilon 1_{\left(x_{N}=g_{u}^{-1}\left(x_{0}^{N}(\epsilon)\right)\right)} \geq \frac{1}{2} .
\end{gathered}
$$

Because $\lim _{N \rightarrow \infty} x_{N}=\infty$, there exists $N_{0}$ such that for all $N>N_{0}$

$$
x_{N}>g_{o}^{-1}\left(x_{0}^{N}(\epsilon)\right) \text { and } x_{N}>g_{u}^{-1}\left(x_{0}^{N}(\epsilon)\right) .
$$

This yields

$$
(1-2 \epsilon)\left(e^{2 x_{0}(\epsilon)}-1\right)-\left(e^{x_{0}(\epsilon)}-e^{-x_{0}(\epsilon)}\right)-1 \geq 0
$$

and $B^{\epsilon}\left(R C S, F_{1}\right)=1.6982 x_{0}(\epsilon)$.

The result for arbitrary $\nu>0$ follows from the scale equivariance of the RCSestimator.

The second estimator presented by Rousseeuw AnD Croux (1993) is the Qestimator

$$
\begin{array}{r}
Q_{N}\left(x_{1}, \ldots, x_{N}\right) \quad:=3 \quad .476\left\{\left|x_{j}\right| ; i<j\right\}_{(l)} \\
\text { with } \quad x_{i}, x_{j}>0 \text { and } l=\left\lceil\frac{\left(\begin{array}{c}
N \\
2
\end{array}\right)}{4}\right\rceil .
\end{array}
$$

Again, the constant 3.476 yields Fisher-consistency for an exponential sample. An asymptotic version of this estimator is given as follows: Let $G \in \mathcal{M}^{1}$ and $X \sim G, Y \sim G$ be stochastically independent. Further let $(X-Y) \sim K_{G}$. Then $Q(G)$ is defined as

$$
Q(G):=3.476 K_{G}^{-1}\left(\frac{5}{8}\right) .
$$

If again $G_{N}$ is the empirical distribution function of $G$, then $Q\left(G_{N}\right)$ is not exactly the same as $Q_{N}$ where one takes an order statistic out of $\left(\begin{array}{l}n \\ 2\end{array}\right)$ elements instead of $n^{2}$, however asymptotically this makes no difference.

Theorem 3.2. The gross-error sensitivity of the Q-estimator is given by

$$
\gamma^{*}\left(Q, F_{\nu}\right)=2.3173 \nu
$$

Proof. The theorem is proven for $\nu=1$. Then the influence function of the Q-estimator equals

$$
I F\left(x ; Q, F_{1}\right)=3.476 \frac{0.25-F_{1}(x+0.2877)+F_{1}(x-0.2877)}{\int f_{1}(y+0.2877) f_{1}(y) d y} .
$$


Substituting $F_{1}(x)=1-e^{-x}, x>0$, and $f_{1}(x)=e^{-x}, x>0$, yields

$$
I F\left(x ; Q, F_{1}\right)= \begin{cases}2.3173, & x \leq-0.2877 \\ -6.952+6.952 e^{-x}, & -0.2877 \leq x \leq 0.2877 \\ 2.3173-5.407 e^{-x}, & x \geq 0.2877\end{cases}
$$

This means

$$
\operatorname{IF}\left(x ; Q, F_{1}\right) \leq 2.3173 \text { for all } x \in \mathcal{R}^{+} .
$$

For arbitrary $\nu>0$, the proposition follows from the scale equivariance of the Q-estimator.

The asymptotic variance of the Q-estimator is

$$
V\left(Q, F_{\nu}\right)=1.3433 \nu^{2}
$$

and the asymptotic relative efficiency is $74 \%$ (RousseEuw AnD CRoux, 1993). In these respects the Q-estimator is comparable with the $10 \%$-trimmed mean $\bar{x}_{10}$ (KIMBER, 1983).

More exactly the gross-error sensitivity of the trimmed mean is $\gamma^{*}\left(\bar{x}_{10}, F_{\nu}\right)=2.1$ and the asymptotic relative efficiency is $A R E\left(\bar{x}_{10}, \hat{\nu}_{M L}\right)=0.85$, but the breakdown point is well known as $\epsilon^{*}\left(\bar{x}_{10}, F_{\nu}\right)=0.1$, while the breakdown point of the Q-estimator is $\epsilon^{*}\left(Q, F_{\nu}\right)=0.5$. Hence, the Q- and the RCS-estimator have the same breakdown point, which is the highest possible breakdown point for scale equivariant estimators of $\nu$.

The explosion bias curve of the Q-estimator is given by (RoUsSEEUW AND Croux, 1993)

$$
B^{\epsilon}\left(Q, F_{\nu}\right)=3.476 \nu F_{*}^{-1}\left(\frac{5-8 \epsilon+4 \epsilon^{2}}{8(1-\epsilon)^{2}}\right)
$$

where $F_{*}(x)$ is the distribution function of the Laplace distribution with

$$
F_{*}(x):= \begin{cases}\frac{1}{2} e^{x}, & x \leq 0 \\ 1-\frac{1}{2} e^{-x}, & x \geq 0 .\end{cases}
$$

Figure 2 compares the explosion bias curves of the above three robust estimators. It can be noted that the standardized median can be recommended here, because

(i) this estimator is very easy to calculate,

(ii) it has minimal gross-error sensitivity,

(iii) its breakdown point is the highest possible for a scale equivariant estimator, 


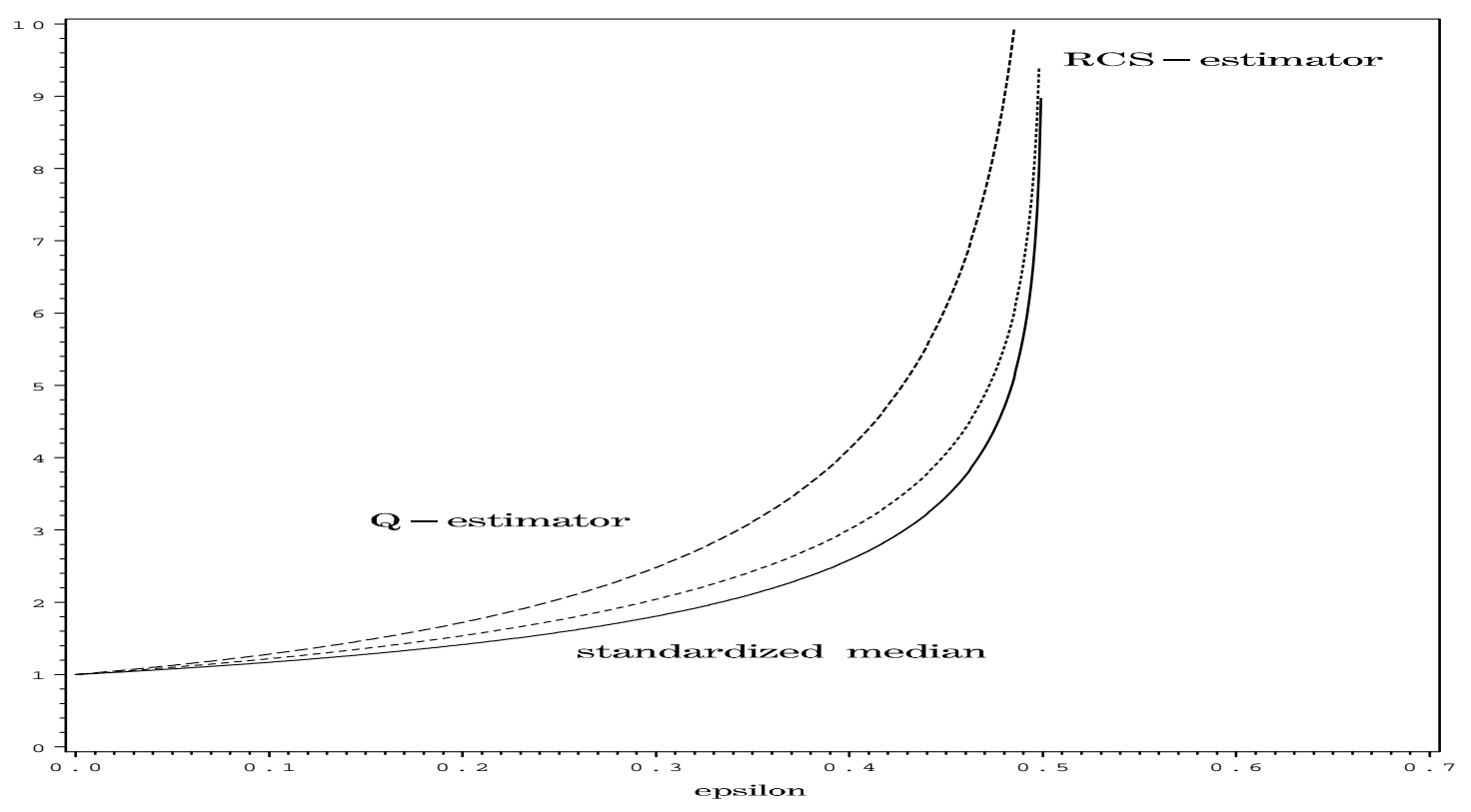

Figure 2: Comparison of the explosion bias curves for $\nu=1$.

(iv) for all $\epsilon \in\left(0, \frac{1}{2}\right)$, the explosion bias curve of the standardized median performs better than the explosion bias curves of the RCS- and the Qestimator.

A comparison of the RCS-estimator and the Q-estimator shows similarly that for all $\epsilon \in\left(0, \frac{1}{2}\right)$ the explosion bias curve of the RCS-estimator takes smaller values than the bias curve of the Q-estimator. Hence, it can be stated that an increase of the asymptotic relative efficiency causes an increase of the gross-error sensitivity which is not surprising.

\section{Acknowledgement}

This work has been supported by the SFB 475 of the DFG.

\section{References}

Donoho, D. L. and LiU, R. C. (1988), The "automatic" robustness of minimum distance functionals, The Annals of Statistics 16, 552-586. 
Hampel, F. R. (1968), Contributions to the theory of robust estimation, Ph. D. Thesis, University of California, Berkeley.

HAMPEL, F. R. (1974), The influence curve and its role in robust estimation, Journal of the American Statistical Association, 69, 383-393.

Hampel, F. R., Ronchetti, E. M., Rousseeuw, P. J. and Stahel, W. A. (1986), Robust statistics - The approach based on influence functions, John Wiley and Sons, New York.

Huber, P. J. (1981), Robust statistics, John Wiley and Sons, New York.

Kimber, A. C. (1983), Comparison of some robust estimators of scale in gamma samples with known shape, Journal of Statistical Computation and Simulation, 18, 273-286.

Rousseeuw, P. J. and Croux, C. (1993), Alternatives to the median absolute deviation, Journal of the American Statistical Association, 88, 1273-1283.

Rousseeuw, P. J. and Leroy, C. (1988), A robust scale estimator based on the shortest half, Statistica Neerlandica 42, 103-116.

Rousseeuw, P. J. and Yohai, V. (1984), Robust regression by means of Sestimators, in: J. Franke, W. Härde and R. D. Martin (eds.), Robust and Nonlinear Time Series Analysis, Lecture Notes in Statistics 26, Springer, New York, 256-272.

Staudte, R. G. and Sheater. S. J. (1990), Robust estimating and testing, John Wiley and Sons, New York. 